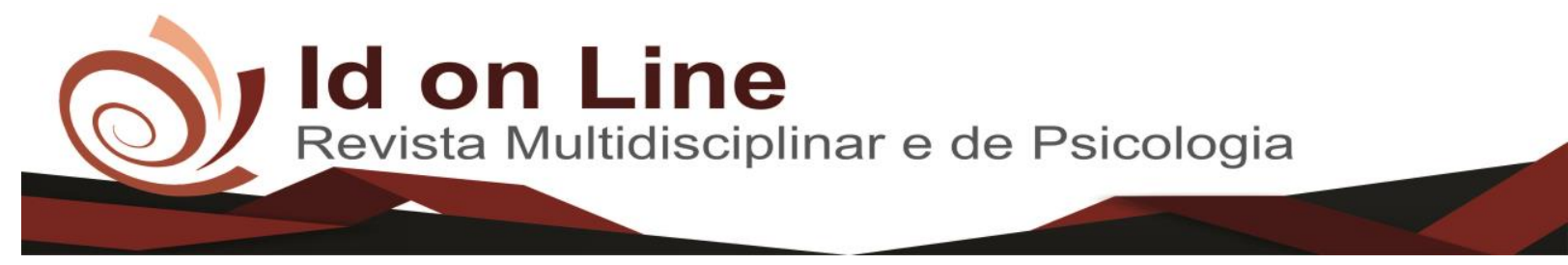

Artigo

\title{
Avaliação da Adesão ao Tratamento Farmacológico de Idosos Portadores de Diabetes Mellitus Tipo II Acompanhados em uma Rede de Farmácias de Vitória da Conquista - Bahia
}

\author{
Sueli Sousa Campos Fernandes ${ }^{1}$; Rodrigo Santos Damascena ${ }^{2}$; Fernanda Santos Portela ${ }^{3}$
}

\begin{abstract}
Resumo: O diabetes mellitus (DM) é considerado uma Doença Crônica Não Transmissível (DCNT) descrita como doença endócrina de caráter multifatorial, representada, principalmente, pela hiperglicemia progressiva e sustentada. Entre os tipos desta patologia, se destaca o diabetes mellitus tipo 2 (DM2) caracterizado por uma resistência insulínica frente às células do corpo e em situações singulares, além da resistência, o portador apresenta um comprometimento na produção do hormônio insulina. Esta pesquisa teve por objetivo avaliar a adesão ao tratamento farmacológico de idosos portadores de diabetes mellitus tipo II, acompanhados em uma rede de farmácias de Vitória da Conquista - Bahia. Tratase de um estudo exploratório e descritivo com abordagem quantitativa. Como instrumento de pesquisa, aplicou-se um questionário estruturado com variáveis sociodemográficas, econômicas e de caráter terapêutico, com base nas escalas de adesão segundo o Teste Morisky e Green. O estudo contou com a participação de 42 idosos, de idade entre 60 e 86 anos, sendo a mais frequente a faixa etária de 60 a 65 anos. O gênero predominante foi o feminino (57\%), 72\% declararam ser pardos e $70 \%$ casados. O teste de Morisky e Green revelou que 52\% dos idosos entrevistados possui alto grau de adesão ao tratamento medicamentoso, em contrapartida $48 \%$ foram classificados com baixa adesão ao tratamento do tipo não intencional (65\%). Os resultados do presente estudo quando avaliados em conjunto observa-se que a adesão é baixa, assim como descrito na literatura. Isso se confirma quando se destaca a população estudada, pois, o idoso já se encontra no processo de alterações fisiológicas e metabólicas próprias do envelhecimento, o que proporciona a ele uma série de limitações, exigido muitas vezes mudanças repentinas, em especial no estilo de vida, tornando-o mais propenso a falhas no manejo da doença. Intensificar os estudos sobre adesão ao tratamento do DM2 e outras DCNT, talvez seja a saída para a promoção da saúde, prevenção ou retardo das complicações e especialmente a melhoria significativa da qualidade de vida do indivíduo.
\end{abstract}

Descritores: Diabetes Mellitus tipo 2; Adesão; Tratamento Farmacológico; Idoso.

\section{Evaluation of Adhesion to Pharmacological Treatment of Older Carriers of Diabetes Mellitus Type II Accompanied in a Network of Pharmacies of Victory of the Conquest - Bahia}

\begin{abstract}
Diabetes mellitus (DM) is considered a chronic noncommunicable disease (CNCD) described as a multifactor endocrine disease, mainly represented by progressive and sustained hyperglycemia. Among the types of this pathology, diabetes mellitus type 2 (DM2) is characterized by an insulin resistance to the cells of the body and in singular situations, besides the resistance, the carrier presents a compromise in the production of the hormone insulin. The objective of this research was to evaluate adherence to the pharmacological treatment of elderly patients with diabetes mellitus type II, followed in a pharmacy network in Vitória da Conquista - Bahia. This is an exploratory and descriptive study with a quantitative approach. As a research instrument, a structured questionnaire was applied with sociodemographic, economic and therapeutic variables, based on the adherence scales according to the Morisky and Green Test. The study had the participation of 42 elderly people, aged between 60 and 86 years, being the highest frequency in the age group of 60 to 65 years. The predominant gender was female (57\%), 72\% declared to be brown and 70\% married. The Morisky and Green test revealed that $52 \%$ of the elderly interviewed had a high degree of adherence to drug treatment. On the other hand, $48 \%$ were classified as having low adherence to unintentional treatment $(65 \%)$. The results of the present study when evaluated together show that adhesion is low, as described in the literature. This is confirmed when the
\end{abstract}

${ }^{1}$ Graduanda em Farmácia pela Faculdade Independente do Nordeste - FAINOR E-mail: suelisousacf@hotmail.com;

${ }^{2}$ Graduação em Ciências Farmacêuticas pela Universidade Estadual de Feira de Santana. Mestre em Saúde Pública pela Fiocruz. Especialista em Microbiologia Clínica. Especialista em Saúde Pública com Ênfase em PSF. Especialista em Gestão da Assistência Farmacêutica. Especialista em Farmácia Clínica e Hospitalar.

${ }^{3}$ Graduação em Farmácia pela Faculdade Independente do Nordeste - FAINOR. Especialista em Análises Clínicas e Toxicológicas. 
population studied is highlighted, since the elderly are already in the process of physiological and metabolic changes typical of aging, which gives him a series of limitations, often requiring sudden changes, especially in lifestyle , making it more prone to disease management failures. Intensifying studies on adherence to treatment of DM2 and other CNCDs may be the way out for health promotion, prevention or delay of complications and especially the significant improvement of the quality of life of the individual.

Keywords: Diabetes Mellitus type 2; Accession; Pharmacological Treatment; Old man.

\section{Introdução}

Nas últimas décadas o processo de globalização se expandiu em todo o mundo e em reflexo a esta causa, inúmeras transformações ocorreram no perfil demográfico, social e econômico, favorecendo, principalmente, o crescimento populacional e o aumento da expectativa de vida. Entretanto, as mudanças na estância epidemiológica também foram afetadas, e consequentemente, as Doenças Crônicas Não Transmissíveis (DCNT) aumentaram de maneira significativa nos últimos anos (VANZELLA; et al, 2018).

As DCNT abrangem um grupo de patologias que proporcionam ao portador experiências clínicas ao longo do seu curso, pois não apresentam cura. Dessa forma, as DCNT são consideradas um problema de saúde pública, pois o índice de morbimortalidade é elevado. Em 2013, os dados da Organização Mundial de Saúde (OMS) demonstravam que as mortes por DCNT chegavam há $63 \%$ no mundo e 72,6\% no Brasil (SILVA; et al. 2017; ABREU; et al., 2017).

O diabetes mellitus (DM) é considerado uma DCNT descrita como doença endócrina de caráter multifatorial, representada, principalmente, pela hiperglicemia progressiva e sustentada. De acordo a Sociedade Brasileira de Diabetes (SBD), em 2014, essa doença acometeu mais de 387 milhões de pessoas no mundo. E em 2040, acredita-se que a DM possa alcançar 642 milhões de pessoas (OTÁVIO; DAMASCENO; LEMOS, 2014; COSTA; et al., 2016; MAIA; COSTA; SILVA, 2017).

O DM pode ser dividido, primordialmente, em tipo 1, tipo 2 e diabetes gestacional, a depender da sua fisiopatologia. O diabetes mellitus tipo 1 (DM1) é mais frequente em crianças e jovens e ocorre de forma abrupta, quando as células beta pancreáticas sofrem deterioração e não produzem o hormônio insulina. O diabetes mellitus gestacional (DMG) é um distúrbio metabólico que ocorre na fase gestacional e é impulsionada pela elevação dos hormônios contrarreguladores da insulina, como por exemplo, o lactogênico placentário, prolactina e progesterona (PADILHA; et al., 2017; MASSUCATTI; PEREIRA; MAIOLI, 2012).

O diabetes mellitus tipo 2 (DM2) é caracterizado por uma resistência insulínica frente às células do corpo. Entretanto, existem situações singulares que além da resistência, o portador de DM2 apresenta um comprometimento na produção do hormônio. O DM2 abrange de 90 a 95\% dos casos de DM e acomete indivíduos de todas as idades e gêneros, contudo, possui maior prevalência entre a população idosa (TESTON; et al., 2017). 
A propensão dos idosos ao DM2 pode ser justificada pelo processo fisiológico do envelhecimento, que promove diversas alterações nos sistemas anatômicos, como a redução do funcionamento cognitivo, metabólico e imunológico. Além disso, a diminuição da capacidade motora impossibilita a realização de grandes esforços, tornando o idoso limitado a desempenhar atividades físicas, o que implica na qualidade de vida e propõe efeitos negativos ao tratamento e controle da doença (MEDEIROS; et al., 2014; CAMPOLINA; et al., 2011).

Quando não tratado e acompanhado adequadamente, o DM2 promove diversos prejuízos ao portador. As complicações mais comuns causadas pela descompensação da glicemia são a retinopatia, nefropatia e neuropatia periférica, que quando se instalam, provocam reparos irreversíveis, e para manejo e estabilização do dano estabelecido, é desprovido elevado impacto econômico ao portador e sua família (COSTA; et al., 2017; SILVA; SOUZA, 2017). A estimativa mundial de gastos anuais para tratar DM se aproxima dos 153 milhões de dólares, e essas despesas podem chegar a 396 milhões em 2025 (ZANGULO; et al.; 2017).

A base para prevenção e controle do DM2 varia desde uma alimentação saudável, prática de exercícios físicos, monitorização da glicemia até o uso contínuo de hipoglicemiantes, que deve ser inserido quando esgotadas as demais medidas de prevenção e controle (SOUZA; et al., 2017). Dessa forma, a adesão terapêutica é fundamental para melhoria dos níveis glicêmicos e manutenção fisiológica, e exige compromisso e fidelidade do portador (FARIA; et al., 2014; FIGUEIRA; et al., 2017).

De acordo com o exposto, essa pesquisa teve por objetivo avaliar a adesão ao tratamento farmacológico de idosos portadores de diabetes mellitus tipo II, acompanhados em uma rede de farmácias de Vitória da Conquista, município brasileiro do estado da Bahia. Este estudo possui grande relevância no que diz respeito à inexistência de registros atuais sobre o tema e suas consequências no município, o que pode auxiliar os gestores a subsidiar decisões financeiras. Além disso, pode ajudar a orientar os profissionais e os futuros profissionais de saúde para acompanhamento do portador de DM2, o que é totalmente pertinente, haja vista a expansão desta doença.

\section{Materiais e Métodos}

Trata-se de um estudo exploratório e descritivo com abordagem quantitativa, realizado em Vitória da Conquista, município brasileiro do estado da Bahia. Este município se localiza na região econômica do Sudoeste baiano, sendo a quinta maior economia do estado e ocupa a posição de terceira maior cidade da Bahia, região Nordeste do Brasil (KOCHERGIN; PROIETTI; CÉSAR, 2014).

O presente estudo foi realizado com idosos portadores de Diabetes Mellitus tipo 2, em uma rede de farmácias composta por três lojas que se localizam em bairros distintos da cidade e abrangem públicos diferentes. Os participantes da pesquisa foram os clientes da rede de farmácias com idade igual ou 
superior a 60 anos, de ambos os sexos, que realizam tratamento medicamentoso para condição clínica em estudo e que seguem ou não as orientações não farmacológicas.

A coleta de dados foi realizada por meio de entrevista direta com os portadores de DM2, de modo individual em uma sala privada do estabelecimento. Como instrumento de pesquisa foi utilizado um questionário estruturado com questões contemplando variáveis importantes para o desenvolvimento deste estudo, como dados socioeconômicos, demográficos, fatores relacionados à terapia e ao paciente e questões específicas com base nas escalas de adesão segundo Teste Morisky e Green.

O teste proposto por Morisky e Green tem como objetivo estimar o nível de adesão ao tratamento farmacológico, considerando aderente ao tratamento o paciente que obtenha pontuação máxima (04 pontos), ou seja, todas as respostas negativas (alto grau de adesão) e, não aderentes, os pacientes que obtiveram 03 pontos ou menos (baixo grau de adesão), (ELIAS, 2011). Esta avaliação também possibilita distinguir o tipo de comportamento do indivíduo frente ao baixo grau adesão, podendo ser classificado como intencional ou não intencional, porém, podem existir pacientes que se comportam com ambos os tipos.

O baixo grau de adesão intencional compreende as respostas afirmativas de ao menos um dos seguintes quesitos: "Quando se sente bem, às vezes, deixa de tomar os seus medicamentos?” ou "Quando se sente mal, às vezes, deixa de tomar seus medicamentos?”. Já no caso do baixo grau de adesão não intencional, se refere à afirmação de no mínimo uma das perguntas: "Às vezes, esquece de tomar seus medicamentos?"ou "Às vezes, se descuida quanto ao horário de tomar seus medicamentos?".

A amostra obtida durante a pesquisa reflete o período de coleta compreendido entre os meses de agosto e setembro de 2018, que possibilitou a participação de 42 idosos de idades entre 60 a 86 anos.

O projeto de pesquisa foi submetido ao Comitê de Ética da Faculdade Independente do Nordeste - FAINOR, atendendo aos aspectos éticos dispostos nas Resoluções no 466/2012 e n 510/2010 do Conselho Nacional de saúde, sendo aprovado pelo parecer $n^{\circ}$ 2.786.796. Os participantes envolvidos da pesquisa receberam um documento denominado Termo de Consentimento Livre e Esclarecido (TCLE), que assegura a integridade do trabalho e esclarecem quaisquer dúvidas acerca do trabalho abordado.

\section{Resultados e Discussão}

A pesquisa contou com a participação de 42 idosos com faixa etária entre 60 e 86 anos, de ambos os sexos. As características sociodemográficas e econômicas estão representadas na Tabela 1. 
Tabela 1. Características sociodemográficas e econômicas dos portadores de diabetes mellitus tipo II, entrevistados em uma rede de farmácias de Vitória da Conquista, Bahia.

\begin{tabular}{|c|c|c|}
\hline \multirow[t]{2}{*}{ Variáveis } & \multicolumn{2}{|c|}{ Frequência } \\
\hline & $\mathbf{N}$ & $\%$ \\
\hline \multicolumn{3}{|l|}{ Gênero } \\
\hline Masculino & 18 & 43 \\
\hline Feminino & 24 & 57 \\
\hline \multicolumn{3}{|l|}{ Faixa etária } \\
\hline 60 a 65 anos & 13 & 31 \\
\hline 66 a 70 anos & 11 & 26 \\
\hline 71 a 75 anos & 12 & 29 \\
\hline 76 a 80 anos & 4 & 9 \\
\hline 81 a 86 anos & 2 & 5 \\
\hline \multicolumn{3}{|l|}{ Cor ou raça } \\
\hline Branco (a) & 9 & 21 \\
\hline Preto (a) & 2 & 5 \\
\hline Pardo (a) & 30 & 72 \\
\hline Não declarou & 1 & 5 \\
\hline \multicolumn{3}{|l|}{ Estado civil } \\
\hline Solteiro (a) & 1 & 2 \\
\hline Casado (a) / Mora com companheiro (a) & 30 & 70 \\
\hline Viúvo (a) & 7 & 19 \\
\hline Separado (a) / divorciado (a) / desquitado (a) & 4 & 9 \\
\hline \multicolumn{3}{|l|}{ Religião } \\
\hline Católica & 29 & 69 \\
\hline Protestante ou evangélico & 11 & 26 \\
\hline Sem religião & 2 & 5 \\
\hline \multicolumn{3}{|l|}{ Onde mora? } \\
\hline Em casa ou apartamento com minha família & 36 & 86 \\
\hline Em casa ou apartamento sozinho (a) & 6 & 14 \\
\hline \multicolumn{3}{|l|}{ Com quem mora? } \\
\hline Mora sozinha (o) & 5 & 12 \\
\hline Esposo (a) / Companheiro (a) & 10 & 24 \\
\hline Filhos (as) & 2 & 5 \\
\hline Outros parentes/ amigos (as) ou colegas & 2 & 5 \\
\hline Esposo (a) / Filhos (as) & 13 & 31 \\
\hline Esposo (a) / Outros parentes/ amigos (as) ou colegas & 3 & 7 \\
\hline Filhos (as) / Outros parentes/ amigos (as) ou colegas & 4 & 9 \\
\hline Esposo (a) / filhos (as) / Outros parentes/ amigos (as) ou colegas & 3 & 7 \\
\hline \multicolumn{3}{|l|}{ Quantas pessoas moram na residência, incluindo o entrevistado? } \\
\hline Mora sozinha (o) & 5 & 12 \\
\hline Duas pessoas & 12 & 28 \\
\hline Três pessoas & 12 & 29 \\
\hline Quatro pessoas & 6 & 14 \\
\hline Cinco pessoas & 5 & 12 \\
\hline Seis pessoas ou mais & 2 & 5 \\
\hline \multicolumn{3}{|l|}{ Escolaridade } \\
\hline Não estudou & 4 & 10 \\
\hline Ensino fundamental $1^{\mathrm{a}}$ a $4^{\mathrm{a}}$ serie (antigo primário) & 24 & 57 \\
\hline Ensino fundamental $5^{\mathrm{a}}$ a $8^{\mathrm{a}}$ serie (antigo ginásio) & 9 & 22 \\
\hline Ensino médio (antigo $2^{\circ}$ grau) incompleto & 1 & 2 \\
\hline Ensino médio completo & 3 & 7 \\
\hline
\end{tabular}




\begin{tabular}{lrc} 
Renda mensal do grupo familiar & 3 & 7 \\
Menos de um salário mínimo & 38 & 91 \\
De um a três salários mínimos & 1 & 2 \\
De três a seis salários mínimos & & \\
Município de residência & 41 & 2 \\
Vitória da Conquista - BA & 1 & 2 \\
Outro município & & 2 \\
Ocupação & & 7 \\
Empregado (a) / Carteira assinada & 1 & 22 \\
Autônomo (a) & 3 & 67 \\
Do lar & 9 & 2 \\
Aposentado (a) / Pensionista & 28 & $\mathbf{1 0 0}$ \\
Desempregado (a) & 1 & $\mathbf{4 2}$ \\
\hline Total
\end{tabular}

Fonte: Dados coletados pela pesquisadora (2018).

A primeira variável analisada foi o gênero dos participantes. Dos 42 idosos entrevistados, $57 \%$ (24) eram representantes do sexo feminino. Este dado é condizente com o estudo de Borba e colaboradores (2018), no qual, foram avaliados os fatores de adesão em idosos portadores de diabetes, e observou-se que dos 150 idosos diabéticos entrevistados, 73,3\% eram do sexo feminino.

O DM2 exige do portador uma infinidade de mudanças comportamentais e fisiológicas. $\mathrm{Na}$ mulher, alguns fatores de risco como, sedentarismo, estresse, obesidade, gestação e climatério podem influenciar diretamente na progressão desta patologia. Além disso, estes números também podem estar ligados a maior procura pelo serviço de saúde (SILVA, et al; 2017; LESSMANN; SILVA; NASSAR, 2011; MANÇÚ; ALMEIDA, 2016; EMÍDIO, et al; 2014).

A análise da faixa etária dos participantes deste estudo, demonstrou que 31\% (13) do total de entrevistados possui idade entre 60 a 65 anos, seguido de $29 \%$ (12) com 71 a 75 anos. Esses resultados corrobora com a pesquisa de Dias e colaboradores (2017), que estudaram os desafios da prática do autocuidado do idoso portador de diabetes mellitus tipo 2, onde do total de 14 idosos com idade entre 60 a 89 anos, $86 \%$ estavam na faixa etária de 60 a 79 anos de idade.

O aumento da sobrevida da população trás consigo diversas transformações, as quais repercutem e influenciam no aumento da prevalência de DM, principalmente o tipo 2. No idoso, surgem as alterações fisiológicas e metabólicas próprias do envelhecimento as quais causam limitações, como a redução da pratica de atividade física, dando lugar ao sedentarismo, e em alguns casos aumenta os maus hábitos alimentares que podem levar a obesidade e principalmente ao surgimento do DM2. Outra justificativa para a prevalência do DM2 na faixa etária dos 60 a 75 anos se deve a oportunidade de diagnóstico da doença a partir dos 45 anos, onde o rastreamento se intensifica devido ao maior número de diabéticos nessa faixa de idade (ISER, et al; 2015).

Quanto à cor de pele ou raça, 72\% (30) dos participantes da pesquisa declararam ser pardos. Dados que corroboram com o estudo de Ferraz, Reis e Lima (2017) que investigaram as condições de 
saúde de idosos portadores de Diabetes mellitus e hipertensão arterial sistêmica em uma amostra de 53 pessoas, 64,8\% (35) consideravam sua cor de pele parda, cor proveniente da miscigenação entre brancos e pretos, deste modo sofre influência da cor preta a qual tem sido citada com maior frequência de diabetes. Entretanto, alguns estudos já apontam que não há prevalência de cor para o surgimento do diabetes, brancos e pretos possui frequência bastante parecidas (ISER, et al., 2015).

Analisou-se também o estado civil dos portadores de DM2, obtendo como resultado que $70 \%$ (30) do total de entrevistados relataram que moram com esposo (a) ou companheiro (a). Esse resultado está de acordo com o estudo de Assunção e colaboradores (2017), que verificou conhecimento e atitude de pacientes com diabetes mellitus da Atenção Primária à Saúde, obteve participação de 354 pessoas com DM2, sendo que $60 \%$ desses eram casados.

Para o indivíduo diabético morar com um parceiro (a) pode trazer efeitos positivos e negativos, no que diz respeito à adesão ao tratamento, ter alguém que auxilia na administração dos medicamentos, dando uma maior atenção aos horários, por exemplo, possibilita uma maior probabilidade de aderir à terapia. No entanto, as cobranças do parceiro ao portador da doença, para que este mude os hábitos de vida, alimentação e comportamento diante da doença podem acarretar no aumento do estresse e sucessivamente no descontrole glicêmico, dificultando o cuidado com o DM2 (ZANCHETTA, et al; 2016).

Outra variável sociodemográfica analisada foi à religião, onde a presente pesquisa apontou que $69 \%$ (29) dos entrevistados declararam ser católicos. Esse dado condiz com o estudo de Santos e Faro (2018), que estudaram a autoeficácia, lócus de controle e adesão ao tratamento em pacientes com diabetes tipo 2, entrevistaram 55 portadores de DM2, destes 72,7\% (40) eram católicos.

Segundo Duarte e Wanderley (2011), a religião possui uma grande importância na vida do idoso, principalmente, sob circunstâncias de agravos a saúde, pois, auxilia no enfrentamento das situações estressantes provenientes da doença, subtendendo uma maior probabilidade de adesão à terapia.

Quanto a variável escolaridade verificou-se nesse estudo que $57 \%$ (24) dos participantes possuem baixa escolaridade, apenas o ensino fundamental de $1^{\mathrm{a}}$ a $4^{\mathrm{a}}$ serie (antigo primário), dados condizentes com o estudo sobre prevalência e fatores associados ao diabetes em idosos, realizado por Vitoi e colaboradores (2015), onde dos 621 idosos entrevistados, 64\% relataram ter estudado até as séries iniciais do ensino fundamental.

O grau de escolaridade é de grande relevância no controle de qualquer doença, principalmente as DCNT. No manejo com o DM2 não é diferente, quanto maior o nível educacional do portador, mais facilidade na busca por informações, maior conhecimento sobre a patologia, e melhor acesso aos programas destinados ao acompanhamento e controle do diabetes. Entretanto, o que revela diversos estudos é que a maioria dos idosos com a doença possui baixa escolaridade, o que dificulta no cuidado com a doença e na prevenção das complicações, visto que a compreensão sobre a doença acaba sendo prejudicada (CORTEZ, et al; 2015). 
A renda do grupo familiar também teve destaque nesse estudo, 91\% (38) dos entrevistados declararam que a renda mensal é de um a três salários, considerando o salário mínimo atual no valor de R\$ 954,00, sendo que, 67\% (28) são aposentados ou pensionistas. A pesquisa realizada por Silva, Fonseca e Rossi (2015) sobre adesão ao tratamento medicamentoso entre pessoas com diabetes mellitus, confirma esse resultado, ao relatar que das 142 pessoas entrevistadas, 60,6\% referiram renda de até 3 salários mínimos, salientam ainda que o baixo poder aquisitivo interfere de forma negativa no tratamento do DM, pois, limita o acesso a informações e as tecnologias que favorecem o controle da doença.

Tabela 1.1. Características sociodemográficas e econômicas dos portadores de diabetes mellitus tipo II, entrevistados em uma rede de farmácias de Vitória da Conquista, Bahia.

\begin{tabular}{lcc}
\hline Variáveis & \multicolumn{2}{c}{ Frequência } \\
\cline { 2 - 3 } & $\mathbf{N}$ & $\mathbf{\%}$ \\
\hline É fumante? & 0 & 0 \\
Sim & 42 & 100 \\
Não & & \\
Faz uso de bebidas alcoólicas & 8 & 19 \\
Sim & 34 & 81 \\
Não & & \\
Realiza atividade física (pelo menos $\mathbf{0 3}$ vezes na semana)? & 17 & 40 \\
Sim & 25 & 60 \\
Não & & \\
& & 74 \\
Faz dieta para controlar o diabetes? & 31 & 24 \\
Sim & 10 & 2 \\
Não & 1 & $\mathbf{1 0 0}$ \\
Ãs vezes & $\mathbf{4 2}$ \\
Total & & \\
\hline
\end{tabular}

Fonte: Dados coletados pela pesquisadora em 2018.

A tabela 1.1 descreve as características sociodemográficas referentes ao estilo de vida e comportamento social dos portadores de DM2. Nesse quesito os participantes foram indagados quanto ao tabagismo, e 100\% (42) da amostra disseram ser não fumantes. Esse resultado possui um importante impacto positivo, no que se refere ao cuidado com a doença, visto que, não fumar contribui tanto na prevenção quanto no retardo do DM. Já para os portadores de DM2, não fumar significa um melhor controle e tratamento da doença, a redução das complicações, impedindo que ocorram, por exemplo, as amputações de membro as quais tem como principal fator de risco, o habito de fumar (ALMEIDA, et al; 2018).

Ao serem questionados sobre o uso de bebidas alcoólicas, $81 \%$ (34) responderam que não utiliza, em contrapartida uma parcela de 19\% (8) dos participantes afirmaram fazer uso de álcool. No estudo de Luz e colaboradores (2015) o percentual de idosos que não usam bebidas alcoólicas também prevaleceu 85,6\% (363), considerando o total de 424 participantes da pesquisa, que verificou o perfil sociodemográfico e de hábitos de vida da população idosa. 
O presente estudo possui fator positivo quanto ao hábito de ingerir álcool, pois, o resultado obtido denota que a maioria dos entrevistados possui clareza quanto aos prejuízos do álcool. O uso do álcool acarreta em inúmeros malefícios a saúde do idoso e ao considerar que este já possua uma doença crônica como o diabetes, esse agravo à saúde aumenta significativamente, afetando o controle glicêmico, por produzir inúmeras interações como o medicamento, reduzindo seu efeito, por exemplo. Ademais estudos mostram que assim como o uso do tabaco, a ingestão de bebidas alcoólicas favorece o surgimento das complicações relacionadas ao DM2 (SILVA, et al; 2017).

A prática de atividade física por no mínimo três vezes durante a semana, também foi analisada nesta pesquisa, sendo que 60\% (25) pessoas responderam que não fazem nenhum tipo de exercício e $40 \%$ (17) dos entrevistados declararam que faz algum tipo de atividade. $\mathrm{O}$ trabalho de Pimenta e colaboradores (2015) que verificou os fatores associados a doenças crônicas em idosos atendidos também revelou que dos 385 entrevistados, apenas 23,9\% praticavam atividade física, demonstrando assim, que o sistema de saúde precisa montar estratégias para manter em atividade o portador de DM2.

A atividade física é fundamental para a melhoria da qualidade de vida do portador de DM2. É uma forte aliada na prevenção das complicações provenientes do DM, quando realizada de forma contínua, ordenada e orientada por um profissional habilitado que irá buscar de forma individualizada a atividade mais adequada, favorecendo a redução da glicemia de jejum e da hemoglobina glicada e ainda melhora a sistema vascular. A inatividade física é um fator preocupante, pois, à medida que o sedentarismo se instala as complicações surgem, a qualidade de vida é reduzida, os maus hábitos alimentares ganham espaço e os distúrbios metabólicos se exacerbam, dificultando o processo terapêutico do paciente (KOLCHRAIBER, et al; 2018; SANTOS; SOUSA; BARROS, 2018 ).

Os participantes foram questionados, se faziam dieta para controlar o diabetes, e 74\% (31), responderam sim a esse quesito. Assim como no estudo de Franco Junior, Heleno e Lopes (2013), que dos 120 pacientes entrevistados, 85,8\% (103) também confirmaram que faz dieta para controlar o diabetes. Esse estudo buscou verificar a qualidade de vida e controle glicêmico do paciente portador de diabetes mellitus tipo 2 .

Manter o controle metabólico é o objetivo no tratamento do diabetes e a terapia não medicamentosa, que envolve alimentação adequada e a prática de atividade física deve ser uma das primeiras opções neste caso, pois aliar ao tratamento uma boa alimentação permite além da redução do nível glicêmico e da hemoglobina glicada, uma melhora significativa na qualidade de vida do diabético, mais do que fazer dieta ou apenas restringir alguns alimentos, o portador de DM2, deve buscar melhorar a alimentação como um todo, adequar o paladar a alimentos saudáveis, sem deixar de sentir sabor (VILLAS BOAS, et al; 2011). 
Tabela 2. Adesão ao tratamento medicamentoso, com base no teste de Morisky e Green.

\begin{tabular}{lcc}
\hline Variáveis & \multicolumn{2}{c}{ Frequência } \\
\cline { 2 - 3 } & $\mathbf{N}$ & $\mathbf{\%}$ \\
\cline { 2 - 3 } Ás vezes se esquece de tomar seus medicamentos? & 15 & 36 \\
Sim & 27 & 64 \\
Não & & \\
Ás vezes se descuida quanto ao horário de tomar de seus & & \\
medicamentos? & 13 & 31 \\
Sim & 29 & 69 \\
Não & & \\
Quando se sente bem, às vezes, deixa de tomar seus medicamentos? & & \\
Sim & 6 & 14 \\
Não & 36 & \\
& & \\
Quando se sente mal com os medicamentos, às vezes, deixa de tomá- & & 7 \\
los? & 3 & $\mathbf{1 0 0}$ \\
Sim & 39 & \\
Não & $\mathbf{4 2}$ & \\
Total & & \\
\hline
\end{tabular}

Fonte: Dados coletados pela pesquisadora em 2018.

A tabela 2 apresenta questões específicas do teste de Morisky e Green que avalia a adesão ao tratamento medicamentoso do DM2, através do comportamento do paciente para com seus medicamentos e tratamento, e se fundamenta na teoria de que o uso incorreto dos medicamentos acontece por: esquecimento, descuido com horário, interrupção do medicamento quando se sente bem ou interromper o medicamento quando se sente mal (SOUSA, et al; 2014).

Quanto ao uso inadequado dos medicamentos por esquecimento, 64\% (27) dos entrevistados declaram não se esquecer de tomar os medicamentos, seguido de $36 \%$ (15) que relataram esquecer. Esses dados são condizentes com os resultados do trabalho de Bezerra, Brito e Costa (2016), o qual contou com 134 idosos, onde a maioria também não se esquece de ingerir os medicamentos $(66,1 \%)$, e $31,4 \%$ que disseram esquecer só às vezes, além 2,5\% que sempre esquecem.

O presente estudo assim como os dados da literatura traz resultados positivos quanto à adesão, pois demonstraram boa atividade cerebral do idoso, que geralmente fica comprometida pelo processo de envelhecimento, causando alterações nas funções cognitivas, como o esquecimento. Entretanto é importante ressaltar que por ser uma resposta subjetiva o idoso pode superestimar sua resposta fazendoa tornar-se verdadeira mesmo que não seja (GAUTÉRIO-ABREU, et al; 2015).

Quanto ao descuido com horário obteve-se um resultado positivo, 69\% (29) dos entrevistados disseram que não se descuidam dos horários da medicação, contra 31\% (13) que não seguem o horário corretamente. No estudo de Estancial e Marini (2013), elas constataram que dos 50 pacientes estudados, $68 \%$ declararam que não se descuidam com o horário dos medicamentos. Entretanto, neste mesmo estudo, as autoras resaltam que o descuido com o horário de administrar o medicamento tenha sido o segundo motivo da não adesão entre os entrevistados, assim como também apontam outros autores, a 
saber: Ramos, Carvalho Filha e Silva (2015), declaram que no estudo deles houve um predomínio de $54,4 \%$ dos usuários que se descuidam do horário de tomar seus medicamentos, bem como, Scherer, Weber e Oliveira (2011) observou em suas pesquisas que 50\% dos entrevistados não seguem o horário determinado.

Portanto apesar do presente trabalho demonstrar resultados positivos, é possível perceber novamente a influência da subjetividade nas respostas, já que vários estudos denotam contrariedade a este. Desconhecer a importância do uso contínuo dos anti-hiperglicemiantes para o controle do DM2, inclusive de seguir o mesmo horário são fatores bastante comuns. O paciente deve ser o principal interessado no controle de sua patologia, assim sendo, é necessário que ele conheça sobre sua doença, saiba suas particularidades para, deste modo se conectar com o tratamento, e entender que assim como o excesso de medicamento é prejudicial, sua falta também traz consequências para sua condição de saúde. (SOLBIATI, et al; 2018).

No quesito, interrupção do uso medicamentos por estarem se sentido bem, $86 \%$ dos participantes responderam não, ou seja, mantém o uso dos medicamentos sem interrupções. Assim como quando questionados sobre deixar de tomar os fármacos quando estão se sentindo mal, 93\% (39) disseram que continuam tomando seus medicamentos também nessa situação. Esse resultado corrobora com o estudo de Silva e colaboradores (2015), onde $84,1 \%$ dos entrevistados responderam que não deixam de tomar o medicamento quando estão se sentido bem e $90,5 \%$ continuam a tomar quando sentem alguma piora.

Apesar dos resultados descritos anteriormente terem sido positivos, pois os participantes do estudo negaram interromper o tratamento nas duas hipóteses levantadas, essas podem trazer inconformidades por serem questões auto-informativas que podem sofrer omissão, falha de memória ou insegurança frente ao entrevistador. Ademais são questões que baseiam em comportamento do indivíduo, sendo classificadas como intencional, e ao considerar a maioria dos estudos que indicam bom predomínio de respostas negativas a esses questionamentos, denota que o portador de DM2 realmente tem se preocupado em seguir o tratamento corretamente (STEFANO, et al; 2017).

Tabela 3: Grau de adesão ao tratamento farmacológico dos entrevistados baseado no teste de Morisky e Green.

\section{Grau de Adesão}

\begin{tabular}{lcc} 
& & $\mathbf{\%}$ \\
\cline { 2 - 3 } & $\mathbf{N}$ & 52 \\
Alto grau de adesão & 22 & 48 \\
Baixo grau de adesão & 20 & \\
& & 65 \\
Tipos de comportamento relacionados ao baixo grau de adesão & 13 & 5 \\
Baixa adesão não intencional & 1 & 30 \\
Baixa adesão intencional & 6 & $\mathbf{1 0 0}$ \\
Baixa adesão com ambos & $\mathbf{4 2}$ & \\
Total
\end{tabular}

Fonte: Dados coletados pela pesquisadora em 2018 
O Teste de Morisky descreve ainda o grau de adesão ao tratamento, classificando com alto grau de adesão as pessoas que tiveram todas as respostas da Tabela 2 negativas, e com baixo grau de adesão àquelas que possuírem ao menos uma resposta afirmativa, estes últimos ainda podem ser classificados quanto ao comportamento podendo ser intencional ou não intencional, ou ambos, como demonstra a Tabela 3.

A análise da Tabela 3 permitiu verificar que 52\% (22) dos entrevistados possui alto grau de adesão, ou seja, responderam todas as questões negativas, em contrapartida $48 \%$ (20) dos participantes responderam ao menos uma questão afirmativa e assim, de acordo com o teste de Morisky e Green possuem baixo grau de adesão. A pesquisa realizada por Medtler e Perassolo, (2016), possui grandes semelhanças com o presente estudo, pois nela $50 \%$ dos participantes foram classificados como aderentes e a outra metade como não aderente.

Com base na literatura é possível verificar que a maioria dos estudos já realizados sobre adesão ao tratamento por meio do teste de Morisky, se destaca a baixa adesão. E considerando a possibilidade de margem de erro neste estudo, temos um empate entre aderentes e não aderentes, tendo em vista a proximidade dos resultados, onde 52\% são aderentes e $48 \%$ não aderentes. Britto e colaboradores, (2015), encontrou 82.7\% não aderentes e apenas 17,2 aderentes e Ramos, Carvalho Filha e Silva (2015), apontou que dos 356 participantes, 67,3\% não aderirem ao tratamento medicamentoso.

É importante ressaltar também, que os fatores que podem levar a essa baixa adesão são complexos, é na verdade uma junção de todas as variáveis citadas neste estudo, desde as características sociodemográficas e econômicas ao comportamento e estilo de vida do paciente. O idoso se torna mais propenso a não adesão, pois a maioria possui polifarmácia, o que dificulta sua relação com o tratamento farmacológico corretamente, assim como com o tratamento não farmacológico, que depende inteiramente do seu querer e compreensão (BRITTO, et al., 2015; RAMOS, CARVALHO FILHA, SILVA, 2015).

Quando relaciona a análise ao tipo de comportamento que levou ao baixo grau de adesão a maior porcentagem é de baixa adesão do tipo não intencional com 65\% (13), seguida dos dois tipos de comportamento (intencional e não intencional) com 30\% (6). Essa classificação se baseia também na Tabela 2, onde as duas primeiras perguntas quanto ao esquecimento e descuido do horário são comportamentos de caráter não intencional e as duas últimas, quanto à interrrupção do tratamento por se sentir melhor ou por se sentir pior são de caráter comportamental intencional. No estudo de Baldoni e colaboradores (2016), o resultado referente à maior porcentagem do tipo não intencional corrobora com os resultados encontrados nesse trabalho, nele, $65 \%$ dos participantes possui baixa adesão não intencional, enquanto $21 \%$ são do tipo intencional.

A baixa adesão não intencional pode ser em decorrência das mudanças proveniente do envelhecimento, onde o estado de alerta e as funções cognitivas sofrem deterioração ao longo dos anos de vida do idoso, isso faz com que ele esqueça ou se descuide do horário de tomar seus medicamentos. Por esse motivo é essencial o apoio familiar no tratamento do DM2 e também o acompanhamento 
farmacoterapêutico, para que as limitações do envelhecer não prejudiquem sua qualidade de vida e o controle de suas patologias crônicas (ISER, et al; 2015; DIAS, et al; 2016).

Tabela 4. Fatores relacionados à terapia dos portadores de diabetes mellitus tipo 2 acompanhados em uma rede de farmácias no interior da Bahia.

\begin{tabular}{lcc}
\hline Variáveis & \multicolumn{2}{c}{ Frequência } \\
& N & \% \\
\cline { 2 - 3 } & & \\
Há quanto tempo sabe que é diabético? & 9 & 22 \\
Menos de 03 anos & 13 & 31 \\
De 03 a 06 anos & 6 & 14 \\
De 06 a 09 anos & 9 & 21 \\
De 09 a 12 anos & 5 & 12 \\
Mais de 12 anos & & \\
Tem dificuldades em ingerir os comprimidos? & 2 & 5 \\
Sim & 40 & 95 \\
Não & & \\
Tem algum sintoma indesejado ao ingerir o medicamento? & 7 & 17 \\
Sim & 35 & 83 \\
Não & $\mathbf{4 2}$ & $\mathbf{1 0 0}$ \\
Total
\end{tabular}

Fonte: dados coletados pela pesquisadora em 2018.

A tabela 4 refere-se ao tempo de diagnóstico do DM2 e também aos fatores relacionados da terapia medicamentosa dos portadores de diabetes. Na análise quanto ao tempo que receberam o diagnóstico 31\% (13) dos 42 entrevistados possui entre três a seis anos que foram diagnosticados, seguido de $22 \%$ (9) que possui menos de três anos de diagnóstico e apenas $12 \%$ (5) dos participantes tinham mais de doze anos de diagnóstico. No estudo de Cortez e colaboradores (2015), 28,2\% (372) dos participantes eram diabéticos há menos de 5 anos, 35,2\% (464) pessoas tinham 5 e 10 anos de doença e $36,6 \%$ (484) pessoas eram diabéticas há mais de 10 anos, resultados que apresentam semelhança com o presente estudo.

É possível observar na literatura que a população idosa é a que possui maior tempo de diagnóstico de diabetes, corrobora com os dados que de após os 45 anos de idade aumentam as possibilidades de diagnóstico, pois o rastreio a partir dessa idade se torna maior, além disso, o idoso devido às alterações que surgem tende a procurar mais os serviços de saúde, detectando a doença mais cedo (ISER, et al; 2015; ANUNCIAÇÃO, et al; 2012).

Analisou-se também nesse estudo se o diabético tinha dificuldade de ingerir seus comprimidos, 95\% (40) responderam que não ter dificuldade, favorecendo assim a adesão ao tratamento. A disfagia é algo comum presente na vida do idoso, é um problema que pode prejudicar a aceitação ao tratamento medicamentoso oral em forma de comprimidos ou drágeas, pois, dificulta a deglutição, trazendo incômodo para o idoso, o qual pode até abandonar a terapia (BERTOTTI, et al; 2015). 
Quanto aos sintomas indesejados o maior percentual de participantes 83\% (35) declararam não sentir nenhum efeito adverso proveniente do uso do medicamento. Entretanto o estudo realizado por Jannuzzi e colaboradores (2014), aponta que as reações adversas são comuns, durante o uso dos antidiabéticos orais, o que pode prejudicar a adesão ao tratamento, pois, influencia no comportamento e crenças do paciente frente ao tratamento.

Tabela 5. Fatores relacionados aos portadores de diabetes mellitus tipo 2 acompanhados em uma rede de farmácias no interior da Bahia.

\begin{tabular}{|c|c|c|}
\hline \multirow[t]{2}{*}{ Variáveis } & \multicolumn{2}{|c|}{ Frequência } \\
\hline & $\mathbf{N}$ & $\%$ \\
\hline \multicolumn{3}{|c|}{ Acredita que os medicamentos são fundamentais para controlar o diabetes? } \\
\hline Nunca & 2 & 4 \\
\hline Quase nunca & 2 & 5 \\
\hline Quase sempre & 10 & 24 \\
\hline Sempre & 26 & 62 \\
\hline Não sabe & 2 & 5 \\
\hline \multicolumn{3}{|c|}{ Acredita nos efeitos positivos do tratamento para o diabetes? } \\
\hline Nunca & 2 & 5 \\
\hline Quase nunca & 1 & 2 \\
\hline Muitas vezes & 1 & 2 \\
\hline Quase sempre & 5 & 12 \\
\hline Sempre & 31 & 74 \\
\hline Não sabe & 2 & 5 \\
\hline \multicolumn{3}{|l|}{ Preocupa-se em medir o diabetes? } \\
\hline Nunca & 4 & 9 \\
\hline Quase nunca & 8 & 19 \\
\hline Muitas vezes & 5 & 12 \\
\hline Quase sempre & 13 & 31 \\
\hline Sempre & 12 & 29 \\
\hline \multicolumn{3}{|c|}{ Possui outra doença além do diabetes? } \\
\hline Sim & 34 & 81 \\
\hline Não & 8 & 19 \\
\hline \multicolumn{3}{|c|}{ Onde adquire / compra a maioria dos medicamentos? } \\
\hline Público & 11 & 26 \\
\hline Privado & 8 & 19 \\
\hline Ambos & 22 & 52 \\
\hline Abandonou o tratamento (não usa) & 1 & 3 \\
\hline \multicolumn{3}{|c|}{ Quantos medicamentos usam para o tratamento do diabetes? } \\
\hline Um medicamento & 26 & 62 \\
\hline Dois medicamentos & 13 & 31 \\
\hline Três medicamentos & 1 & 2 \\
\hline Nenhum (abandonou tratamento) & 2 & 5 \\
\hline \multicolumn{3}{|c|}{ Quantos medicamentos usam para outras patologias? } \\
\hline Um & 7 & 17 \\
\hline Dois & 13 & 31 \\
\hline Três & 5 & 12 \\
\hline Quatro & 6 & 14 \\
\hline Cinco & 3 & 7 \\
\hline Seis & 2 & 5 \\
\hline Sete & 2 & 5 \\
\hline Nenhum & 4 & 9 \\
\hline Total & 42 & 100 \\
\hline
\end{tabular}

Fonte: Dados coletados pela pesquisadora em 2018. 
A tabela 5 apresenta análises realizadas sobre os portadores de DM2, suas crenças e preocupações acerca do tratamento, analisando também a existência de comorbidades e da polifarmácia.

A primeira análise demonstrou que $62 \%$ (26) do total de diabéticos entrevistados acreditam que os medicamentos são fundamentais para controlar o diabetes, assim como 74\% (31) dos participantes acreditam nos efeitos positivos do tratamento para o diabetes. Esse resultado corrobora com o estudo de Borba e colaboradores (2018), que destaca que a crença do idoso sobre o uso dos medicamentos no tratamento do DM2 é positiva, e ainda destaca a importância de considerar essa avaliação de comportamento e credibilidade do portador de diabetes no processo de adesão, pois, tais comportamentos influenciam diretamente na resposta terapêutica e controle da doença.

Quando questionados sobre se preocuparem em automonitorar a glicemia capilar, 31\% (13) responderam que quase sempre verificam sua glicemia e $29 \%$ (12) estão sempre preocupados com seus níveis glicêmicos. Segundo estudo de Veras e colaboradores (2014), que avaliou o autocuidado dos pacientes com a monitorização da glicemia capilar, dos 74 entrevistados, 45,3\% automonitoram a glicemia com uma frequência de até quatro dias na semana e 53,4\% faz essa automonitorização de 5 a 7 dias na semana. Esse resultado corrobora o presente estudo, tendo em vista que a maioria dos participantes da pesquisa respondeu se preocuparem com monitorar a glicemia capilar.

A monitorização da glicemia em portadores de DM2 em uso de antidiabéticos orais e principalmente que usam a insulina, pode ser uma aliada fundamental, tanto para verificação dos níveis glicêmicos quanto na adesão ao tratamento, visto que saber como estão seus níveis glicêmicos, torna o paciente assíduo ao autocuidado para manter o controle da doença. Incentivar a automonitorização da glicemia, principalmente para o idoso, é um desafio que deve ser considerado e aperfeiçoado para fazer parte da rotina do paciente diabético, os resultados tendem a ser positivos para o tratamento do DM2 (FELIX, et al., 2015).

Analisando a presença de comorbidades, verificou-se que $81 \%$ (34) dos entrevistados possui outra doença, além do diabetes, sendo a hipertensão arterial e a dislipidemia as doenças mais citadas. Esse resultado corrobora com o estudo de Cecílio e colaboradores (2015), que apontam que dos 318 diabéticos entrevistados, $66,4 \%$ são também hipertensos e 37,7\% possui hipercolesterolemia.

A hipertensão arterial sistêmica (HAS) é muito comum entre os portadores de DM2, podendo ser explicada, segundo Pinho e colaboradores (2015), principalmente por fatores fisiológicos e patológicos do DM, como a resistência insulínica, estresse oxidativo e as alterações provocadas pela hiperglicemia no sistema renina-angiotensina-aldosterona são alguns dos fatores que favorecem o surgimento da HAS no diabético. No caso da dislipidemia o risco do diabético desenvolver é maior, pois a resistência à insulina provoca alterações no metabolismo dos lipídios e proteínas circulantes. Ademais é importante salientar que qualquer tipo de comorbidade no diabetes é um fator preocupante por favorecer o surgimento das complicações e também exigir do indivíduo maiores cuidados.

Quanto à aquisição dos medicamentos 52\% (22) dos entrevistados disseram que adquirem seus medicamentos em ambos os setores, público e privado, em contrapartida, 26\% (11) declararam que 
conseguem seus medicamentos no setor público. Entretanto segundo estudo de Bezerra, Brito e Costa (2016), 52,9\% dos entrevistados adquire seus medicamentos no setor público e 36,4\% adquire em ambos os setores, demonstrado assim dados controversos a este estudo.

A dificuldade de conseguir os medicamentos no SUS se torna um empecilho ao usuário que prefere pagar para obter seus medicamentos com maior comodidade e liberdade, entretanto, alguns idosos que necessitam de polifarmácia, devido às comorbidades, se esforçam para procurar por serviços públicos para obter seus medicamentos (MATTA, et al., 2018).

Analisou-se também neste estudo a quantidade de medicamentos utilizados para o tratamento do DM2, 62\% (26) do total de idosos entrevistados responderam que utilizam apenas um medicamento para o tratamento do DM, seguido de 31\% (13) que utilizam dois tipos e ainda 5\% (2) que relataram ter abandonado o tratamento. Esse resultado possui semelhanças com o estudo de Lima e colaboradores (2015) que avaliou as interações medicamentosas potenciais em diabéticos do tipo 2, constatando que dos 71 participantes do estudo $36 \%$ estavam em uso de apenas um medicamento e 38,9\% usavam mais de um medicamento.

Diferente do resultado apresentado nesse estudo, à literatura evidência que ocorre um predomínio de polifarmácia no tratamento do DM, principalmente entre idosos, isso se explica pela diabetes descompensada, surgimento de complicações cada vez mais frequentes e também de outras comorbidades, interferindo na adesão, pois, muitas vezes, ocorre o aumento das doses dos medicamentos e o idoso tem dificuldade de se adequar a essas alterações ou a uma nova terapia e também o risco de interações e de sintomas indesejados é maior, o que pode levar até mesmo ao abandono do tratamento (SILVA, et al., 2018).

Quanto ao uso de outros medicamentos para o tratamento das demais doenças, 31\% (13) usam dois medicamentos, seguido de $17 \%$ (7) que usam um. O resultado é condizente com o trabalho de Bezerra, Brito e Costa (2016), onde observou que a maioria dos idosos participantes fazia uso de dois a cinco tipos de fármacos, totalizando $70,8 \%$ dos entrevistados, enquanto $11,3 \%$ usavam de seis a nove tipos de medicamentos.

A polifarmácia do idoso tem sido cada vez mais observada, somente nesse estudo com apenas 42 participantes, analisando os resultados da Tabela 5 já se verifica a quantidade de medicamentos utilizados, pois, a maioria como já mencionado possui além do diabetes alguma outra patologia, e por isso, o número de medicamentos usados diariamente varia entre 3 e 6 fármacos diferentes. Isso pode implicar negativamente no tratamento tanto do DM2 quanto das outras doenças, dificultando a adesão, por erro de doses, esquecimento, interações medicamentosas entre outros (AMES, et al., 2016). 
Tabela 6: Medicamentos utilizados para tratar o diabetes mellitus tipo 2 dos portadores acompanhados em uma rede de farmácias no interior da Bahia.

\begin{tabular}{lcc}
\hline Medicamentos & \multicolumn{2}{c}{ Frequência } \\
\cline { 2 - 3 } & $\mathbf{N}$ & $\mathbf{\%}$ \\
\hline Metformina & 23 & 55 \\
Glibenclamida & 1 & 3 \\
Glicazida & 1 & 3 \\
Metformina + Glibenclamida & 3 & 7 \\
Metformina + Glicazida & 6 & 14 \\
Metformina +Alogliptina + Glicazida & 1 & 2 \\
Metformina +Glibenclamida + insulina & 1 & 2 \\
Metformina + Insulina & 1 & 2 \\
Linagliptina & 2 & 5 \\
Insulina & 1 & 2 \\
Nenhum medicamento & 2 & 5 \\
Total & $\mathbf{4 2}$ & $\mathbf{1 0 0}$ \\
\hline
\end{tabular}

Fonte: Dados coletados pela pesquisadora em 2018.

A Tabela 6 destaca a análise realizada acerca dos medicamentos utilizados no tratamento do DM2, observa-se a prevalência da monoterapia com a metformina a qual é utilizada por 55\% (23) dos entrevistados, seguida da associação de metformina e glicazida 14\% (6) e de metformina e glibenclamida 7\% (3). Esse resultado quanto ao uso da metformina corrobora com a pesquisa realizada por Ames e colaboradores (2016), que ao analisar a classe de antidiabéticos orais usados pelos pacientes diabéticos, destacaram-se as Biguanidas (metformina) 82,6\% seguido de Sulfonamidas (glibenclamida) $72,4 \%$.

A metformina é a primeira escolha no tratamento do DM2 justificando sua prevalência nesse e em outros estudos. Pertencente à classe das Biguanidas, a metformina reduz a liberação hepática de glicose e aumenta sua captação nos tecidos periféricos levando assim a diminuição dos níveis glicêmicos. Entretanto, este fármaco possui muitos efeitos adversos, principalmente, gastrointestinais, o que prejudica muito na adesão ao tratamento. Atualmente a metformina é comumente utilizada em associação com as Sulfonilureias (exemplo: glibenclamida e glicazida), hipoglicemiantes secretagogos que aumentam a secreção de insulina, medicamento eficaz na redução da glicemia (ALVES DA CONCEIÇÃO, NOGUEIRA DA SILVA E BARBOSA, 2017).

\section{Considerações Finais}

Este estudo buscou associar uma diversidade de variáveis sociodemográficas, econômicas e de caráter terapêutico cujo objetivo foi avaliar a adesão ao tratamento farmacológico dos idosos portadores de DM2, com o intuito de auxiliar os gestores a subsidiar decisões financeiras, prezando pela qualidade e eficiência na prestação de serviços de saúde a população, além de orientar os profissionais e os futuros 
profissionais de saúde no acompanhamento do idoso diabético, o que é totalmente pertinente, haja vista a expansão da doença.

Os resultados do presente estudo, se analisados separadamente, podem ser considerados razoáveis quanto ao quesito de maior interesse, a adesão ao tratamento farmacológico. Entretanto, quando avaliado o conjunto de fatores que estão inter-relacionados observa-se que esse resultado de adesão (52\%) é baixo assim como descrito na literatura. Isso se confirma ainda mais quando se destaca a população estudada, pois, o idoso já se encontra no processo de alterações fisiológicas e metabólicas próprias do envelhecimento, o que proporciona a ele uma série de limitações, exigido muitas vezes mudanças repentina, em especial no estilo de vida, tornando-o mais propenso a falhas no manejo da doença.

Como foi observado, por exemplo, quando avaliado o tipo de comportamento relacionado à baixa adesão, ocorreu uma prevalência da baixa adesão não intencional, que esta associada ao esquecimento e descuido de horário dos medicamentos para o tratamento do DM2. Haja vista que esse tipo de comportamento provém das alterações cognitivas que surgem naturalmente com o envelhecer. Ademais o idoso tende a diminuir a função motora, a qual limita a prática de atividade física, que também prejudica o tratamento não farmacológico, forte aliado para uma adesão completa ao tratamento e controle do DM2.

Verificando essa junção de fatores que proporcionam uma boa adesão é importante ressaltar que aderir ao tratamento do diabetes não significa apenas utilizar o medicamento de maneira correta, mais sim, associar o uso correto do fármaco com uma alimentação adequada e a prática de atividade física (no mínimo três vezes na semana).

Ademais intensificar os estudos sobre adesão ao tratamento das DCNT, principalmente as mais comuns como Diabetes e Hipertensão, talvez seja a saída para a promoção da saúde, prevenção ou retardo das complicações e especialmente a melhoria significativa da qualidade de vida do indivíduo.

\section{Referências}

ABREU, S., S., S., DE; OLIVEIRA, A., G., DE; MACEDO, M., A., S., S; DUARTE, S., F., P; REIS, L., A., DOS; LIMA, P., V; Prevalência de Doenças Crônicas não Transmissíveis em Idosos de uma Cidade do Interior da Bahia. Id on Line Revista Multidisciplinar e de Psicologia, v. 11, n. 38. 2017.

ALMEIDA, F., DAS, C., A., DE; COSTA, M., M., L; BASTOS, R., A., A; ALMEIDA, R., A., DE; PEQUENO, G., A; BRILHANTE, E., A., DE, A. Idosos diabéticos: fatores clínicos predisponentes para amputação de membros inferiores. Revista Nursing, v. 21, n. 238, p. 2075-2079, 2018.

ALVES DA CONCEIÇAO, R; NOGUEIRA DA SILVA, P; BARBOSA, M., L.,C. Fármacos para o Tratamento do Diabetes Tipo II: Uma Visita ao passado e um Olhar para o Futuro. Rev. Virtual Quim., v. 9,n. 2, p. 514-534, 2017. 
AMES, K., DA, S; BASSANI, P., H; MOTTER, N; RORATTO,B; HAMMES, J., L., N; QUADRO, M., DO, N., DE; LISSARASSA, Y., P., S; FRIZZO, M., N; ZIMMERMANN, C., E; VIERA,E.,K. Avaliação de hipertensos e diabéticos usuários de polimedicação em santo Ângelo/RS. Revista Saúde Integrada, v. 9, n. 17, p. 58-65, 2016.

ANUNCIAÇÃO, P., C; BRAGA, P., G; ALMEIDA, P., S., DE; LOBO, L., N., PESSOA, M., C. Avaliação do conhecimento sobre alimentação antes e após intervenção nutricional entre diabéticos tipo 2. Revista Baiana de Saúde Pública, v.36, n.4, p. 986-1001, 2012.

ASSUNÇÃO, S., C; FONSECA, A., P; SIlVEIRA, M., F; CALDEIRA, A., P; PINHO, L., DE. Conhecimento e atitude de pacientes com diabetes mellitus da Atenção Primária à Saúde. Escola Anna Nery, Revista de Enfermagem, v. 21, n. 4, p. 1-7, 2017.

BALDONI, N., R; OLIVEIRA, R., E., M., DE; FRANCO, L., J; DAL FABBRO, A., L. Adesão ao tratamento farmacológico de pessoas com diabetes mellitus tipo 2. Rev. Bras. Farm. Hosp. Serv. Saúde, v.7, n.4, p. 15-19, São Paulo, 2016.

BERTOTTI, M., E., Z; SOUZA, A., R., DE; ALMEIDA, D., V., DE; SEDA, J., M; POPIM, R., C. Autopercepção da saúde bucal de idosos em interface com doenças crônicas e uso de medicações. Arq Med. Hosp Fac. Cienc. Med. Santa Casa São Paulo, n. 60, p. 54-60, 2015.

BEZERRA, T., A; BRITO M., A., A., DE; COSTA, K., N., DE, F., M. Caracterização do uso de medicamentos entre idosos atendidos em uma unidade básica de saúde da família. Cogitare Enferm, v. 21, n. 1, p. 01-11, 2016.

BORBA, A., K., DE, O., T; MARQUES, A., P., DE O; RAMOS, V., P; LEAL, M., C., C; ARRUDA, I., K., G., DE; RAMOS, R., S., P., DA, DILVA. Fatores associados à adesão terapêutica em idosos diabéticos assistidos na atenção primária de saúde. Ciência \& Saúde Coletiva, v. 23, n. 3, p. 953-961, 2018.

BRITTO, A., S; ABREUB, H., C., DE, A; RUBIRAC, E., A; MARCOND,S., R., OLIVEIRA, J., R., T., DE. Grau de adesão de idosos internados em um hospital ao regime terapêutico para hipertensão e diabetes. Revista de Atenção à Saúde, v. 13, n. 44, p.27-32, 2015.

CAMPOLINA, A., G.; DINI, P., S.; CICONELLI, R., M. Impacto da doença crônica na qualidade de vida de idosos da comunidade em São Paulo (SP, Brasil). Revista Ciência \& Saúde Coletiva, v. 16, n. 6, p. 2919$2925,2011$.

CECILIO, H., P., M; ARRUdA, G., O., DE; TESTON, E., F; SANTOS, A., L; MARCON, S., S. Comportamentos e comorbidades associados às complicações microvasculares do diabetes. Acta Paul Enferm, v. 28, n. 2, p. 113-119, 2015.

CORTEZ, D., N; REIS, I., A; SOUZA, D., A., S; MACEDO, M., M., L; TORRES, H., DE, C. Complicações e o tempo de diagnóstico do diabetes mellitus na atenção primária. Acta Paulista de Enfermagem, v. 28, n. 3, p. 250-255, 2015.

COSTA, A., F.; FLOR, L., S.; CAMPOS, M., R.; OLIVEIRA, A., F.; COSTA, M., F., S.; SILVA, R., S.; LOBATO, L., C., DO P.; SCHARAMM, J., M., A. Carga do diabetes mellitus tipo 2 no Brasil. Cadernos de Saúde Pública, v. 33, n. 2, 2017.

COSTA, J., R., G.; BRITO, F., A., L.; OLIVEIRA, K., S.; OLIVEIRA, M., M., DE; OLIVEIRA, T., F., F.; OLIVEIRA, L., L. Educação em saúde sobre atenção alimentar: uma estratégia de intervenção em enfermagem aos portadores de Diabetes Mellitus. Mostra Interdisciplinar do curso de Enfermagem - Uni Católica, v. 2, n. 1, 2016.

DIAS, A; CUNHA, M; RIBEIRO, O; ALBUQUERQUE, C; ANDRADE, A. Crenças acerca da medicação e adesão terapêutica em utentes hipertensos. Revista Servir, v. 59, n. 3, p. 50-55, 2016. 
DIAS, E., G; PARDIM, A., C., S; ANTUNES, L., P; SILVA, I., O; ALVES, J., C., S; JORGE, S., A. Desafios da prática do autocuidado do idoso portador de diabetes mellitus tipo 2. Revista SUSTINERE, v. 5, n. 1, p. 38-53, Rio de Janeiro, 2017.

DUARTE, F. M; WANDERLEY, K. D. S. Religião e espiritualidade de idosos internados em uma enfermaria geriátrica. Psicologia: Teoria e Pesquisa, v. 27, n. 1, p. 49-53, 2011.

ELIAS, G., DE, L. Avaliação da adesão ao tratamento da Hipertensão Arterial em pacientes do município de Diamante D’Oeste/PR. Dissertação (Mestrado em Farmacologia) - Centro de Ciências Biológicas, Universidade federal de Santa Catarina, Toledo - Paraná, 2011.

EMÍDIO, A., B; MACHADO, A., L., S., L; DE SÁ, F., M., Q; Nascimento, J., A; QUEIROZ, B., B., DE; FREITAS, R., F; ROCHA, J., S., B. COMPARAÇÃO ENTRE AUTOCUIDADO E HIPERTENSÃO EM MULHERES PORTADORAS DE DIABETES MELLITUS TIPO II. Revista Eletrônica Gestão \& Saúde, v. 05, edição especial p.2632-40, 2014.

ESTANCIAL, C., S; MARINI, D., C. Aderência de diabéticos ao tratamento medicamentoso. Foco: Caderno de Estudos e Pesquisas - Ano 4 - nº 5, 2013.

FARIA, H,. T., G.; SANTOS, M., A., DOS; ARREᄀLIAS, C., C., A.; RODRIGUES, F., F., L.; GONELA, J., T.; TEIXEIRA, C., R., DE S.; ZANETTI, M., L. Adesão ao tratamento em diabetes mellitus em unidades da Estratégia Saúde da Família. Revista da Escola de Enfermagem da Universidade de São Paulo, v. 48, n. 2, p. 257-63, 2014.

FELIX, L., G; LEMOS, L., G., H; NEGREIROS, R., V., DE; MEDEIROS, F., DE, A., L; SOARES, M., J., G., DE. Perfil socioeconômico e clínico de um grupo de diabéticos em tratamento hemodialítico em Curitiba. Anais do Congresso Internacional do Envelhecimento Humano- CIEH, v. 2, n.1, 2015.

FERRAZ, M., DE, O., S; REIS, L., A., DOS; LIMA, P., V. Condições de saúde de idosos portadores de Diabetes mellitus e hipertensão arterial sistêmica. Id on Line Rev. Psic. v.10, n. 33, 2017.

FIGUEIRA, A., L., G.; VILlAS BOAS, L., C., G.; COELHO, A., C., M.; FREITAS, M., C., F.; PACE, A., E. Intervenções educativas para o conhecimento da doença, adesão ao tratamento e controle do diabetes mellitus. Revista Latino-Americana de Enfermagem, v. 25, 2017.

FRANCO JUNIOR, A., J., DE, A; HELENO, M., G., V; LOPES, A., P. Qualidade de vida e controle glicêmico do paciente portador de Diabetes Mellitus tipo 2. Revista Psicologia e Saúde, v. 5, n. 2, p. 102108, 2013.

GAUTÉRIO-ABREU, D., P; SANTOS, S., S., C; SILVA, B., T., ILHA, S; GOMES5, G., C. Pessoas idosas em atendimento ambulatorial: motivos que levam a adesão/não adesão aos medicamentos. Texto Contexto Enferm, v. 24, n. 4, p. 1094-103, Florianópolis, 2015.

ISER, B., P., M; STOPA, S., R; CHUEIRI, P., S; SZWARCWALD, C., L; MALTA, D., C; MONTEIRO, H., O., DA., C; DUNCAN, SCHMIDT, M., I. Prevalência de diabetes autorreferido no Brasil: resultados da Pesquisa Nacional de Saúde 2013. Epidemiol. Serv. Saúde, Brasília, v. 24 n. 2, p. 305-314, 2015.

JANNUZZI, F., F; RODRIGUES, R., C., M; CORNELIO, M., E; SÃO-JOAO, T., M; GALLANI, M., C., B., J. Crenças relacionadas à adesão ao tratamento com antidiabéticos orais segundo a Teoria do Comportamento Planejado. Rev. Latino-Am. Enfermagem, v. 22, n. 4, p. 529-537, 2014.

KOCHERGIN, C., N; PROIETTI, F., A; CESAR, C., C. Comunidades quilombolas de Vitória da Conquista, Bahia, Brasil: autoavaliação de saúde e fatores associados. Cad. Saúde Pública [online], v.30, n.7, p.14871501, 2014. 
KOLCHRAIBER, F., C; ROCHA, J., S; CÉSAR, D., J; MONTEIRO, O,. O; FREDERICO, G., A; GAMBA, M., A. Nível de atividade física em pessoas com diabetes mellitus tipo 2. Rev. Cuid., v. 9, n. 2, p. 21052116, 2018.

LESSMANN, J., C. SILVA, D., M., G., V. DA; NASSAR, S., M. Estresse em mulheres com Diabetes mellitus tipo 2. Rev. Bras Enferm, Brasília v. 64, n. 3, p. 451, 2011.

LIMA, R., F; MACHADO, A., V; REBELO, F., M; NAVES, J., DE, O., S; LAVICH, T., R; DULLIUS, J. Interações medicamentosas potenciais em diabéticos tipo 2 participantes de um programa de educação em saúde. Revista Infarma, v. 27, ed3, p. 160-167, 2015.

LUZ, E., P., DA; DALLEPIANE, L., B; KIRCHNER, R., M; SILVA, L., A., A., DA; SILVA, F., P., DA; KOHLER, J; GOPINGER, E; CARLOT, J., M. Perfil sociodemográfico e de hábitos de vida da população idosa de um município da região norte do Rio Grande do Sul, Brasil. REV. Bras. Geriatr. Gerontol, v. 17, n. 2, p. 303-314, Rio de Janeiro, 2014.

MAIA, M., B.; COSTA, G., S.; SILVA, K., C., F., DA. Associação entre diabetes mellitus e doença periodontal. Revista Intercâmbio, v. 10, 2017.

MANÇÚ, T., DE S; ALMEIDA, O., S., C. Conhecimentos e sentimentos das gestantes diabéticas sobre a diabetes mellitus gestacional e tratamento. Rev. Enferm. UFPE (on line), Recife, v. 10, n.3, p. 1474-82, 2016.

MASSUCATTI, L., A.; PEREIRA, R., A.; MAIOLI, T., U. Prevalência de Diabetes Gestacional em Unidades de Saúde Básica. Revista de Enfermagem e Atenção à Saúde, v. 1, n. 1, 2012.

MATTA, S., R; BERTOLDI, A., D; EMMERICK, I., C., M; FONTANELLA, A., T; COSTA,K., S; LUIZA, V., L; PNAUM, G. Fontes de obtenção de medicamentos por pacientes diagnosticados com doenças crônicas, usuários do Sistema Único de saúde. Cad. Saúde Pública, v. 34, n. 3, 2018.

MEDEIROS, P., A., DE; STREIT, I., A; SANDRESCHI, P., F; FORTUNATO, A., R; MAZO, G., Z. Participação masculina em modalidades de atividades físicas de um Programa para idosos: um estudo longitudinal. Ciênc. saúde coletiva [online], v.19, n.8, p.3479-3488, 2014.

MEDTLER, K; PERASSOLO, M., S. Avaliação da adesão ao tratamento anti-hipertensivo e antidiabético de um grupo hiperdia do município de presidente Lucena - RS. Revista Conhecimento Online, v. 2, n. 8, p. 35-46, $2^{\circ}$ sem. Novo Hamburgo 2016.

OTÁVIO, G., M., DA C.; DAMASCENO, V., DA M., S.; LEMOS, T., N. Importância do Conceito de Medicina Periodontal na Integralidade da Assistência à Saúde. Oral Sciense, v. 6, n. 2, p. 10-17, 2014.

PADILHA, A., P.; ROSA, L., M., DA; SCHOELLER, S., D.; JUNKES, C.; MENDEZ, C., B.; MARTINS, M., M., F., P., DA S. Manual de cuidados às pessoas com diabetes e pé diabético: construção por scoping study. Texto \& Contexto Enfermagem, v. 26, n. 4, 2017.

PIMENTA, F., B; PINHO, L; SILVEIRA, M., F; BOTELHO, A., C., DE, C. Fatores associados a doenças crônicas em idosos atendidos pela estratégia de saúde da família. Revista Ciênc. saúde coletiva, v. 20, n. 8 , 2015.

PINHO, L; AGUiAR, A., P., S; OLIVEIRA, M., R; BARRETO, N., A., P; FERREIRA, C., M., M. Hipertensão e dislipidemia em pacientes diabetes mellitus tipo 2: uma revisão integrativa. Revista Norte Mineira de Enfermagem, v. 4, n. 1, p. 87-101, 2015.

RAMOS, J., S; CARVALHO FILHA, F., S., S; SILVA, R., N., A., DA. Avaliação da adesão ao tratamento por idosos cadastrados no programa do Hiperdia. Revista de Gestão em Sistemas de Saúde - RGSS, v. 4, n. 1, 2015.

SANTOS, C., M., DE, J; FARO, A. Autoeficácia, lócus de controle e adesão ao tratamento em pacientes com diabetes tipo 2. Rev. SBPH, v. 21 n. 1, p. 74-91. Rio de Janeiro, 2018. 
SANTOS, G., M; SOUSA, P., V., DE, L; BARROS, N., V., DOS, A. Perfil epidemiológico dos idosos diabéticos cadastrados no Programa hiperdia no estado do Piauí, Brasil. Rev. Aten. Saúde, v. 16, n. 56, p. 48-53, São Caetano do Sul, 2018.

SCHERER, V., C; WEBER, B., R; OLIVEIRA, K., R., DE. Perfil dos medicamentos utilizados por idosos atendidos em uma farmácia comunitária do município de IJUÍ/RS. Revista Contexto \& Saúde Ijuí, v. 10, n. 20 , p. $375-384,2011$.

SILVA, A., L., DA. FONSECA, G., S., S; ROSSI, V., S., C. Adesão ao tratamento medicamentoso entre pessoas com diabetes mellitus. Revista Ciência et Praxis v. 8, n. 16, 2015.

SILVA, A., P., DA; BORGES, B., V., DE, S; LIRA NETO, J., C., G; AVElinO, F., V., S., D; DAMASCENO, M., M., C; FREITAS, R., W., J., F., DE. Adesão ao tratamento com antidiabéticos orais na atenção básica de saúde. Revista Rene, v. 16, n. 3, p.425-433, 2015.

SILVA, C., S; SANTOS, B., M; ALMEIDA, H., B., O., DE; EL FAHL, L., R; GALVÃO, L., R; GALVÃO, C., R; PORTO,E., C., L; MIRANDA, S., S; FIGUEREDO, A., C., M., G; COELHO, J., M., F. Estilo de vida e condição metabólica de mulheres diabéticas e/ou hipertensas de uma região urbana. Rev. Saúde Col. UEFS, Feira de Santana, v.7, n. 2, p. 65-72, 2017.

SILVA, D., G.; TOSTO, M., C.; EVANGELISTA, M., T.; SILVA, J., N., O.; ABREU, T.; MONTEIRO, C., L., S.; MACEDO, A., C.; SILVA-BOGHOSSIAN, M., DA. Protocolo de atendimento da clinica de periodontia UNIGRANRIO: Pacientes com Diabetes Mellitus. Revista Rede de Cuidados em Saúde, v. 10 n. 1, 2017.

SILVA, E., F; LASTE, G; TORRES, R., L; HIDALGO, M., P., L; STROHER, R; TORRES, I., L., DA, S. Consumo de álcool e tabaco: fator de risco para doença cardiovascular em população idosa do sul do Brasil. Revista Saúde e Desenvolvimento Humano, v. 5, n. 1, p. 23-33, 2017.

SILVA, M., R., R., DA; DINIZ, L., M; SANTOS, J., B., R., DOS; REIS, E., A; MATA, A., R., DA; ARAÚJO, V., E., DE; ÁLVARES, J; ACURCIO, F., DE, A. Uso de medicamentos e fatores associados à polifarmácia em indivíduos com diabetes mellitus em Minas Gerais, Brasil. Ciência \& Saúde Coletiva, v. 23, n.8, p.2565-2574, 2018.

SOCIEDADE BRASILEIRA DE DIABETES. Diretrizes da Sociedade Brasileira de Diabetes 2017-2018. Editora Clannad - p. 23-24. São Paulo, 2017.

SOLBIATI, V., P; OLIVEIRA, N., R., C., DE; TEIXEIRA, C., V., LA, S; GOMES, R., J. Adesão ao tratamento para prevenir agravos relacionados à hipertensão arterial e ao diabetes. Revista Brasileira de Obesidade, Nutrição e Emagrecimento, v.12, n.73, p.629-633. São Paulo, 2018.

SOUSA, D., M., P., DE; SILVA, D., L; FONTENELE, R., P; ARAUJO, P., M; CARVALHO, A., L., M. Métodos indiretos para mensurar a adesão ao tratamento medicamentoso na hipertensão arterial: uma revisão integrativa da literatura. Boletim Informativo Geum, v. 4, n. 1, p. 50-64, 2014.

SOUZA, J., D.; BAPTISTA, M., H., B.; GOMIDES, D. DOS S.; PACE, A., E. Adesão ao cuidado em diabetes mellitus nos três níveis de atenção à saúde. Escola Anna Nery, v. 21, n.4 - P. 1-9, 2017.

STEFANO, I., C., A; CONTERNO, L.,O; SILVA FILHO, C., R., DA; MARIN, M., J., S. Uso de medicamentos por idosos: análise da prescrição, dispensação e utilização num município de porte médio do estado de São Paulo. Rev. Bras. Geriatr. Gerontol, v. 20, n. 5, p. 681-692, Rio de Janeiro, 2017.

TESTON, E., F.; SERAFIM, D.; CUBAS, M., R.; HADDAD, M., DO C., L.; MARCON, S., S. Fatores associados ao conhecimento e à atitude em relação ao Diabetes mellitus. Cogitare Enfermagem, v. 22, n. 4, 2017. 
VANZELLA, E.; NASCIMENTO, J., A., DO; SANTOS, S., R., DOS; O Envelhecimento, a Transição Epidemiológica da População Brasileira e o Impacto nas Hospitalizações. Revista Eletrônica Estácio Saúde, v. 7, n. 1, 2018.

VERAS, V., S; SANTOS, M., A., DOS; RODRIGUES, F., F., L; ARRELIAS, C., C., A; PEDERSOLI, T., A., M; ZANETTI, M., L. Autocuidado de pacientes inseridos em um programa de automonitorização da glicemia capilar no domicílio. Rev. Gaúcha Enferm, v. 35, n. 4, p. 42-48, 2014.

VILLAS BOAS, L., C., G; FOSS, M., C; FOSS-FREITAS, M., C; TORRES, H., DE, C; MONTEIRO, L., Z; PACE, A., E. Adesão à dieta e ao exercício físico das pessoas com Diabetes Mellitus. Texto Contexto Enferm, v. 20, n. 2 p. 272-279. Florianópolis, 2011.

VITOI, N., C; FOGAL, A., S; NASCIMENTO, C., DE, M; FRANCESCHINI, S., DO, C., C; RIBEIRO, A., Q. Prevalência e fatores associados ao diabetes em idosos no município de Viçosa, Minas Gerais. REV BRAS EPIDEMIOL, v. 18, n. 4, p. 953-965, 2015.

ZANCHETTA, F., C; TREVISAN, D., D; APOLINÁRIO, P., P; SILVA, J., B., DA; LIMA, M., H., DE, M. Variáveis clínicas e sociodemográficas associadas com o estresse relacionado ao diabetes em pacientes com diabetes mellitus tipo 2. Revista Einstein, v. 14 n. 3 p. 346-51, 2016.

ZANGUlO, A.; Bari, M., L.; Uceda, M., I. Avaliação de Custo do Tratamento de Doentes com Diabetes Internados no Serviço de Medicina do Hospital do Prenda, Angola. Revista Portuguesa de Farmacoterapia, v. 9 n. 3, p. 154-160, 2017.

\section{Como citar este artigo (Formato ABNT):}

FERNANDES, Sueli Sousa Campos; DAMASCENA, Rodrigo Santos; PORTELA, Fernanda Santos. Avaliação da Adesão ao Tratamento Farmacológico de Idosos Portadores de Diabetes Mellitus Tipo II Acompanhados em uma Rede de Farmácias de Vitória da Conquista - Bahia. Id on Line Rev.Mult. Psic., 2019, vol.13, n.43, p. 241-263. ISSN: 1981-1179.

Recebido: 23/11/2018;

Aceito: $24 / 11 / 2018$ 